\title{
HiCOMEX: Facial Action Unit Recognition Based on Hierarchy Intensity Distribution and COMEX Relation Learning
}

\author{
Ziqiang Shi*1, Liu Liu ${ }^{1}$, Zhongling $\mathrm{Liu}^{1}$, Rujie $\mathrm{Liu}^{1}$, Xiaoyu $\mathrm{Mi}^{2}$, and Kentaro Murase ${ }^{2}$ \\ ${ }^{1}$ Fujitsu Research and Development Center, Beijing, China \\ ${ }^{2}$ Fujitsu Laboratories Ltd., Kawasaki, Japan
}

\begin{abstract}
The detection of facial action units (AUs) has been studied as it has the competition due to the wide-ranging applications thereof. In this paper, we propose a novel framework for the AU detection from a single input image by grasping the co-occurrence and mutual exclusion (COMEX) as well as the intensity distribution among AUs. Our algorithm uses facial landmarks to detect the features of local AUs. The features are input to a bidirectional long short-term memory (BiLSTM) layer for learning the intensity distribution. Afterwards, the new AU feature continuously passed through a self-attention encoding layer and a continuous-state modern Hopfield layer for learning the COMEX relationships. Our experiments on the challenging BP4D and DISFA benchmarks without any external data or pre-trained models yield F1-scores of $63.7 \%$ and $61.8 \%$ respectively, which shows our proposed networks can lead to performance improvement in the AU detection task.
\end{abstract}

\section{INTRODUCTION}

Faces carry a large amount of information about our psychology and emotional states at all time, thus we can quantify mental status based on facial analysis. An action unit, defined by the Facial Action Coding System (FACS) 4, represents the basic facial muscle movement or expression change, and the aim of facial action unit detection is to detect and estimate the occurrence of certain individual facial muscle movements by determining whether the AUs appear or not. AU detection has a vast range of applications, for example, the improvement of labour productivity by taking care of employee's psychology and improving their motivation, or estimating customer's satisfaction and improving their purchasing motivation (digital marketing). In these cases, a detail description of $\mathrm{AU}$ occurrence needs to be estimated from the facial images to complete the subsequent tasks, e.g., facial micro-expression analysis, thus it is a problem that must be solved achieve satisfactory performance in subtle facial signal analysis tasks.

Many techniques based on deep learning have been proposed for this task. Zhao et al. 24 presented deep regional feature learning and multi-label learning modules in a unified architecture for facial AU detection. $\mathrm{Li}$ et al. 9 proposed the use of an EAC-Net that can learn both feature enhancing and region cropping functions simultaneously. Corneanu et al. 3 proposed a deep structured inference network (DSIN) for AU detection in two stages: in the first stage, learned local and global features are combined for initial estimations of AU occurrences; in the second stage, structural inference is used to capture AU relationships by passing information among the initial estimations for final predictions of AU occurrences. Niu et al. [1] improved the AU recognition by using facial shape information extracted from landmarks, although they did not extract a feature map for each AU or utilize any AU relationships. Shao et al. [16, 15] developed an end-to-end framework for joint facial AU detection and face alignment, which can contribute to each other by sharing features and initializing the attention maps with the results of face alignment.

Although these methods are effective, there are still two difficulties in facial AU detection that have not been resolved. Firstly, the subtle change of local facial appearance must be accurately measured to judge whether an AU happens or not: this remains challenging. Individuals may have different levels

*Corresponding author: shiziqiang@cn.fujitsu.com 
of expressiveness due to their physical characteristics, which further increases the difficulty in detecting subtle facial changes. The second shortcoming of previous methods is that they do not fully explore cooccurrence and mutual exclusion (COMEX) of different AUs for overall AU prediction. AUs are not mutually independent of each other, since facial expressions are closely related to AU occurrences [23, 20. As with the examples shown in Fig. 1. some AUs usually happen simultaneously, while some AUs never appear at the same time, which means several AUs can usually be active at the same time and certain AU combinations are more likely to happen than others.

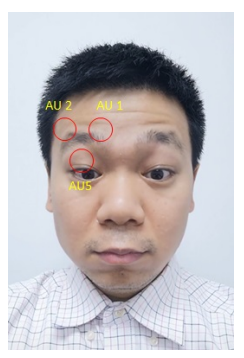

(a) Co-occurrence of AU1, AU2,(b) Co-occurrence of AU4, AU7, and AU5.

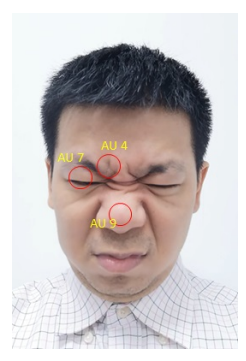
and AU9.

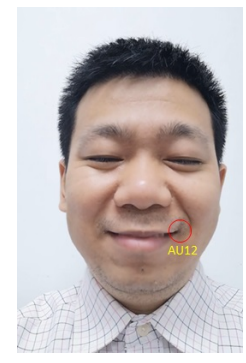

(c) Sample of AU12.

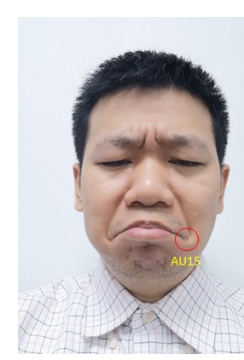

(d) Sample of AU15.

Figure 1: Examples of co-occurrence and mutually exclusive relations between AUs. A surprised expression may cause AU1, AU2, and AU5 to appear together. An angry expression may cause AU4, AU7, and AU9 to appear together. Generally AU12 and AU15 will not appear at the same time.

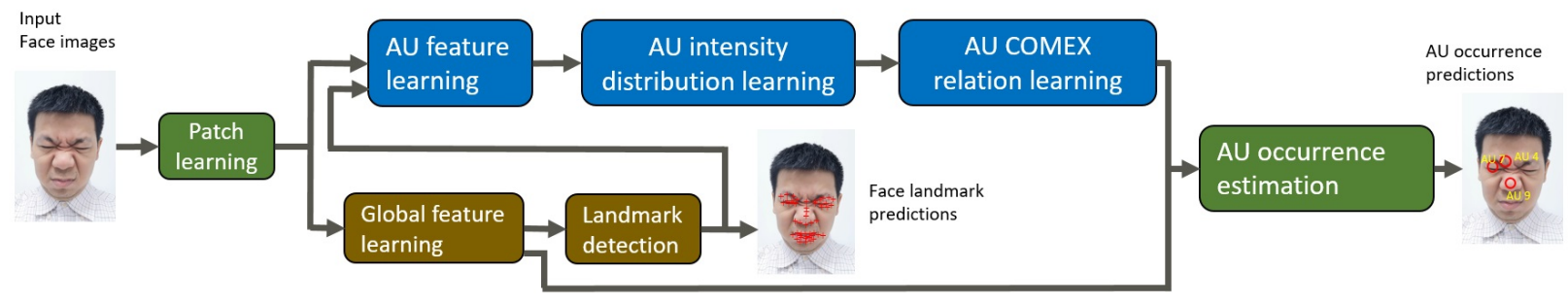

Figure 2: Framework of the proposed method. First, the patch learning module is used to convert the input facial signal into its patch learning feature. Then, the global facial feature and local AU features are extracted based on this patch learning feature. Before concatenating these two features, the AU features are recombined through the interaction among AUs to maximise the characterisation efficacy. The AU occurrences are predicted based on the concatenated representations.

To overcome the above limitations, we propose an end-to-end deep neural network approach called hierarchical local $\mathrm{AU}$ region intensity distribution and COMEX relation learning (HiCOMEX) for AU detection as shown in Fig. 2. HiCOMEX can dynamically calculate the intensity distribution and COMEX relationships among all AUs based on each input image, and strengthen the expressive ability of AU feature maps.

The key contributions of this paper are demonstrated as follows:

- HiCOMEX is the first study to adapt single image to BiLSTM to reflect intensity distribution among AUs into AU feature maps.

- HiCOMEX is the first study to employ the self-attention mechanism and the modern Hopfield network 12 to address the AU COMEX relationship modelling for AU detection. It can use this relationship to enhance the representation ability of $\mathrm{AU}$ features. In other words, these modules naturally make HiCOMEX independent of a person's identity.

- HiCOMEX can achieve state-of-the-art performance on the notable BP4D and DISFA benchmarks. 
The remainder of this paper is organised as follows: Section 2 introduces related work. Section 3 describes our proposed HiCOMEX and AU detection algorithm. The experimental set-up and results are presented in Section 4 . We conclude this paper in Section 5.

\section{Related work}

Our HiCOMEX is closely related to existing deep learning-aided facial AU detection methods as well as AU modelling with relation (graph) learning methods, since we combine both AU detection models and relationship (graph) learning models.

Chu et al. 22 proposed a hybrid deep learning framework that used the strengths of CNNs and LSTMs to model and utilise both spatial and temporal cues. Ertugrul et al. [5] proposed a simple sigmoidal attention mechanism for weighting the local facial patches to detect specific AUs. Li et al. 8 combined the fixed knowledge graph of AU correlation into a convolution neural network to enhance AU detection. Reale et al. 13. extracted local AU features in the 3-d space based on cloud input. Tu et al. [18 implemented an identity-aware architecture of multi-task network cascades where one task is to extract identity information, the other is to subtract that identity information and undertake AU detection. Fan et al. 7 presented a new learning framework that automatically learns the latent relationships between AUs via establishing semantic correspondences between feature maps.

Our proposed HiCOMEX is a more flexible solution that can treat all AU feature maps as a sequence data or a graph data at the same time. We can dynamically calculate the relationship between AUs based on the input image in real-time, and use this relationship to improve the characterisation ability of AU feature maps. In other words, our method is naturally independent of a person's identity.

\section{AU recognition with HiCOMEX}

\subsection{AU Feature Representation Learning}

Figure 3 illustrates the detailed framework of our proposed approach to achieve this goal. Firstly the patch learning module [24] maps a facial image to its latent feature representation called patch features with precise features of local regions. To improve localisation and classification, 'PatchConv' 24] is adopted here for the patch learning. With the PatchConv structure, the initial feature map obtained by 2-d original image is divided spatially into several parts, which are then input separately to the following branches where independent but identical convolution operations are implemented. After that, the feature maps from these branches are reconcatenated to form the final feature map. For details of 'PatchConv' and 'FeatConv', please refer to the work of 24 .

Then inspired by DSIN [3], the global facial feature, local AU features, and facial landmark features are extracted based on patch features. All global facial feature, facial landmark feature, and local AU feature representations are extracted through several layers of 'FeatConv', which consists of 2 Conv2Ds, BatchNorms, PReLUs, and a 2-D max-pooling (Max Pool2D).

\subsection{Local AU Feature Extraction}

In HiCOMEX, local AU features play a more important role than the global counterpart, due to their role in later intensity distribution and COMEX relationship learning and their contribution to final feature representation. In our implementation, the local AU features are not obtained by an extra process, instead, they are cropped and refined from the certain global feature map according to the local facial area of each $\mathrm{AU}$, as shown in Fig. 4. As we know, the facial region which causes the occurrence of each AU is manually defined, e.g., AU1 and AU2 come respectively from muscle movement in the inner brow and outer brow area. With this prior knowledge, we may further define the approximate region for each AU, as illustrated in Fig. 4. The landmark feature will be used to predict the locations of the landmark points. According to the locations of the landmark points, we can roughly determine the facial area corresponding to each AU and will then extract the corresponding AU feature map according to the local area of the face corresponding to the AU. To mine and use the relationship among AUs, all local AU features will pass through an AU intensity distribution learning module and a COMEX relation module to enhance their representation ability, and the 


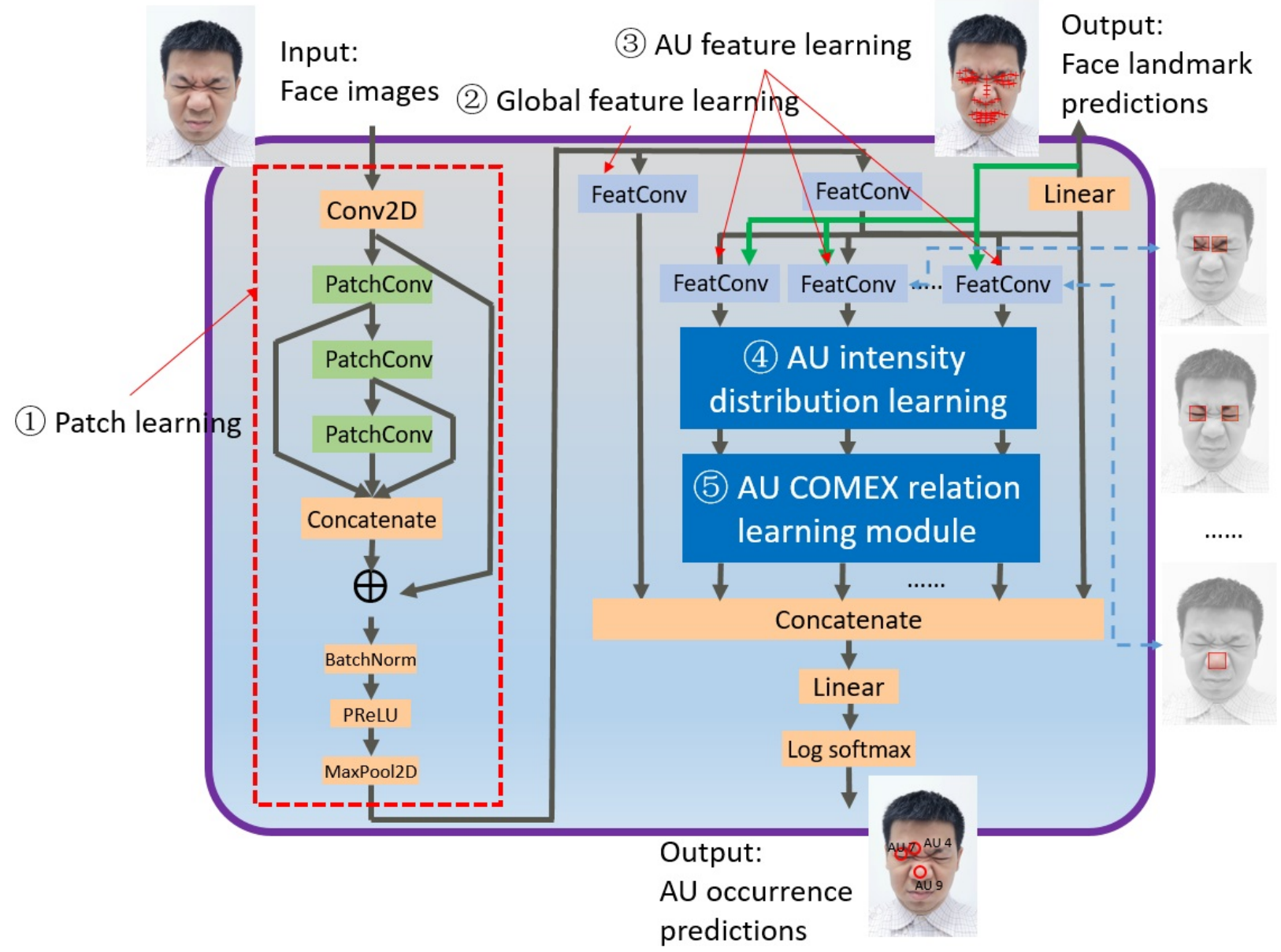

Figure 3: The architecture of HiCOMEX consists of 1) patch learning, 2) global facial feature learning, 3) AU feature learning, 4) AU intensity distribution learning (see Fig. 5), 5) AU COMEX relation learning (see Fig77 6) AU occurrence prediction, and 7) facial landmark estimation.

output contains the refined new local AU features, which will be concatenated with the global facial feature and landmark feature map, for final AU recognition.

\subsection{AU Intensity Distribution Learning}

In this study we adapt single image into BiLSTM to handle intensity distributions resulting from intensity differences among AUs due to the asynchronicity of occurrence of AUs. When a person is adopting a certain expression, due to the physical characteristics of the facial muscles, some AUs usually appear asynchronously. Within a labelled AU data set consisting of many single images, AUs will follow a certain statistical intensity distribution in each $\mathrm{AU}$ co-occurrence and mutual exclusion pattern, therefore, the AU feature maps from a single image must include information of the intensity difference that represents an intensity distribution pattern. We propose to learn this intensity distribution using BiLSTM.

As shown in Fig. 5, we input AU feature maps obtained from a single image by the previous AU feature extraction into BiLSTM as sequence data. Any predefined AU order, such as natural order AU1, AU2, ..., and AU24, can be input into BiLSTM, since knowledge about the order of AUs is unknown or undecided. Bi-directional LSTMs are used to allow information to propagate in both directions to interact the AU feature maps in the sequence back and forth. Intensity distribution learning is data-driven: in one image, the intensities of AUs are different because the AUs occur in different orders. The intensity differences due to the asynchronous AU occurrence can be learned through BiLSTM, while at the same time this method will enhance high-intensity AUs and weaken low-intensity AUs from the viewpoint of classifying the intensity 


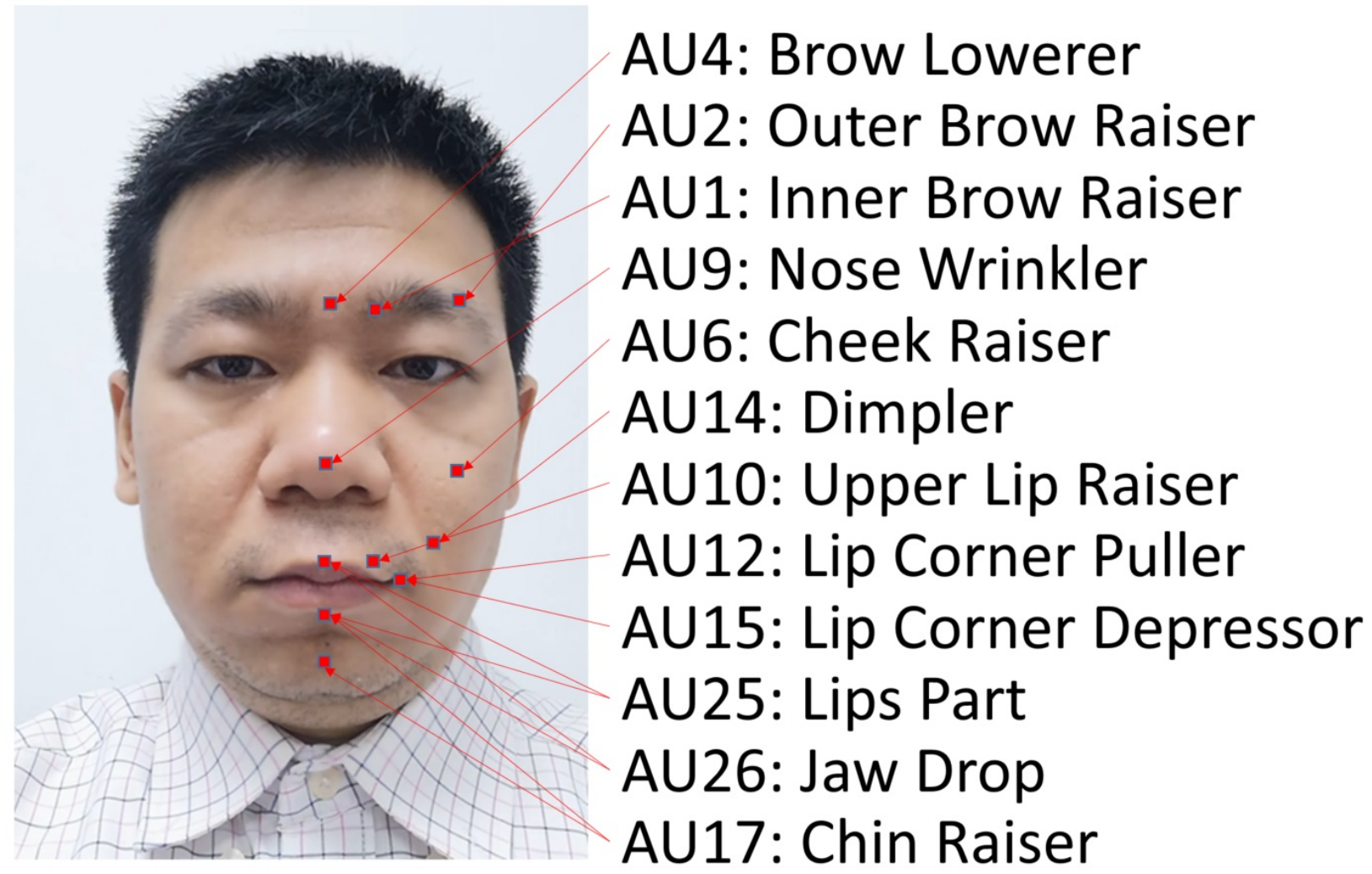

Figure 4: The centers of AUs.

distribution patters. In this study, we adapted each single image to BiLSTM for the first time to reflect the intensity distribution of AUs into AU feature maps. BiLSTM can create the new AU features with intensity distributions to enhance their discriminative ability with regard to AUs.

\subsection{AU COMEX Relation Learning with Self-Attention Encoding}

As described in Section 3.3, the intensity distribution information among AU features is explored by BiLSTM as a sequential structure, however, the COMEX relationship among AUs, which may contribute more to enhancing the discriminative ability of AU features is not involved at all. To alleviate this weakness, a graph structure is naturally adopted to describe such a relationship between AUs [7, 20, 8, .

In the graph structure, each $\mathrm{AU}$ is represented as a graph node, while the relationship such as the probability of co-occurrence among two AUs is represented as the graph edge. The higher the probability that two AUs appear together, the larger the corresponding edge value, and vice versa. For example, the value of the edge that connects AU12 and AU15 should be a very small value, because these two AUs are conflicting and never appear at the same time. In this section we use self-attention [19] to model both the graph and sequence structure of AUs simultaneously.

As shown in Fig. 6, the AU feature map first passes through the pre-processing module, which contains fully connected linear units (Linear), layer normalisation (LayerNorm) [1, dropout (Dropout) [17, and a position encoding module. The position encoding is undertaken as follows

$$
\begin{aligned}
p e(p o s, 2 i) & =\sin \left(\text { pos } / 10000^{2 i / d}\right) \\
p e(p o s, 2 i+1) & =\cos \left(\text { pos } / 10000^{2 i / d}\right)
\end{aligned}
$$

where pos is the position of the AU feature in the input AU graph and $i$ denotes the dimension in the linearlytransformed AU features, and $d$ is the output dimension of the linear component of the pre-processing module. This pe vector is added to the linearly-transformed AU features. After the preprocessing module, we acquire input AU feature maps for the self-attention encoding module. 


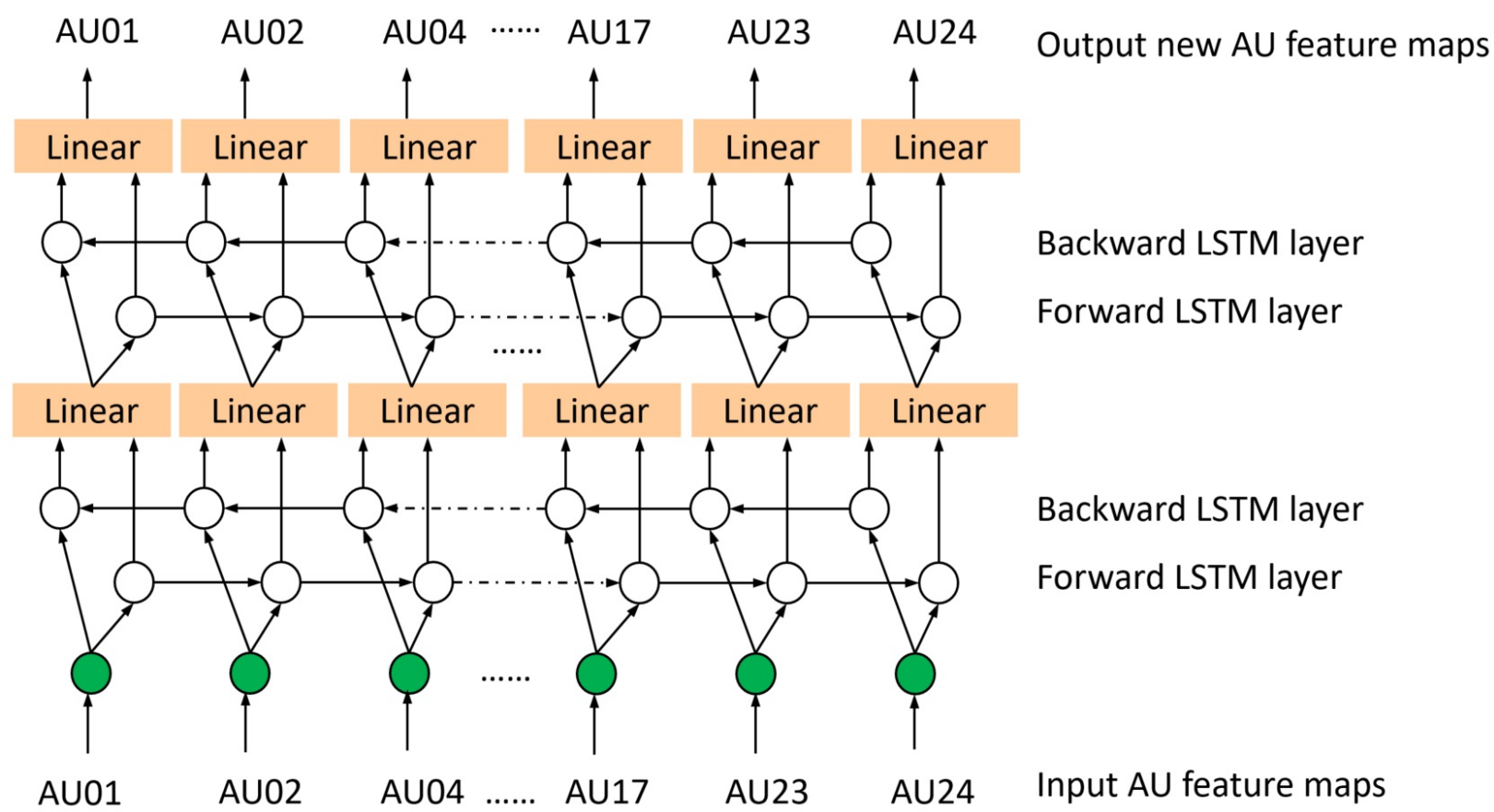

Figure 5: AU intensity distribution learning with BiLSTM. The feature maps corresponding to all AUs are input into BiLSTM as a sequence, and the output is the AU feature maps after recombination and transformation. The reason for using BiLSTM in two directions is to help all AUs before and after can interact and influence with each other.

Several identical self-attention encoding layers can be used in our framework. In each self-attention encoding layer, the input consists of queries and keys of dimension $d_{k}$, and values of dimension $d_{v}$. Here queries, keys, and values are different avatars of the same AU feature map, although modified through different linear transformations. Then we compute the attention function on a set of queries simultaneously, packed together into a matrix $Q$. The keys and values are also packed together into matrices $K$ and $V$. We compute the matrix of outputs as:

$$
\text { Self-Attention }(Q, K, V)=\operatorname{softmax}\left(\frac{Q K^{T}}{\sqrt{d_{k}}}\right) V .
$$

Indeed $\frac{Q K^{T}}{\sqrt{d_{k}}}$ describes the distances between all AU node pairs in the graph. Thus Self-Attention $(Q, K, V)$ is indeed the weighted sum of $\mathrm{AU}$ features according to the relations computed by $\frac{Q K^{T}}{\sqrt{d_{k}}}$, thus result in new AU representations. This means that co-occurring AUs will enhance each other and mutually exclusive AUs will reduce the probability of their co-occurrence.

\subsection{AU COMEX Relation Learning with a Continuous Hopfield Layer}

Recently Ramsauer et al. 12 proposed the use of a modern Hopfield network with continuous states. This novel modern Hopfield network [12] generalises the original Hopfield network with continuous states, which provides a good choice for relationship learning due to its intrinsic graph structure. A simple and effective training method is proposed, where the basic principle of the training method is to obtain the attention by constantly providing queries to the network, akin to the self-attention mechanism. Through this discovery, they have improved the existing self-attention mechanism. Here we also try to use this new model to learn the COMEX relationship between AUs and strengthen the characterisation ability of AU feature maps.

As shown in Fig. 7, the difference in modern Hopfield network from a self-attention system is that the updating formula in Eq. (3.3) can be applied iteratively to the initial state of each Hopfield layer header. After the last update, the new state will be projected to the resulting pattern. Therefore, the Hopfield layer 
allows multiple update steps in a forward direction without changing the number of parameters. The number of update steps per Hopfield header can be specified. In addition, a threshold can be set for the number of updates based on the size of each Hopfield header.

\subsection{Losses}

HiCOMEX has two kinds of outputs (as shown in Fig 3 ), one is AU occurrence prediction, which is a 0/1 prediction for each $\mathrm{AU}$, the other is landmark prediction, which are the coordinates of each landmark. In the present paper, we use two kinds of losses, namely the cross-entropy loss of AU occurrence based on the concatenated global and local feature maps and the Euclidean loss of landmark prediction accuracy based on the landmark feature maps.

\subsection{Two-stage Training}

During the experiment, we found that the two-step training method works better. That is, firstly we train the pipeline in Fig. 3 without the local AU relationship-learning module to acquire the best patch learning, global facial representation learning, and local AU representation learning modules. Then the local AU relation learning module is again added to result in the HiCOMEX, and the parameters the best patch learning, global facial representation learning, and local AU representation learning modules are fixed, only training local AU relationship learning, AU occurrence prediction, and facial landmark estimation modules. The training algorithm we use is SGD, with a momentum of 0.9 and a weight decay of 0.0005 . We train HiCOMEX with an initial learning rate of 0.01 for up to 30 epochs, where the learning rate is multiplied by a coefficient of 0.3 for every two epochs.

Table 1: F1-score(\%) in a comparative study of different state-of-the-art detection methods on the BP4D dataset.

\begin{tabular}{c|c|c|c|c|c|c|c|c|c|c|c|c|c}
\hline \hline AU & 1 & 2 & 4 & 6 & 7 & 10 & 12 & 14 & 15 & 17 & 23 & 24 & Avg \\
\hline \hline LSVM & 23.2 & 22.8 & 23.1 & 27.2 & 47.1 & 77.2 & 63.7 & 64.3 & 18.4 & 33.0 & 19.4 & 20.7 & 35.3 \\
JPML & 32.6 & 25.6 & 37.4 & 42.3 & 50.5 & 72.2 & 74.1 & 65.7 & 38.1 & 40.0 & 30.4 & 42.3 & 45.9 \\
DRML & 36.4 & 41.8 & 43.0 & 55.0 & 67.0 & 66.3 & 65.8 & 54.1 & 33.2 & 48.0 & 31.7 & 30.0 & 48.3 \\
CPM & 43.4 & 40.7 & 43.3 & 59.2 & 61.3 & 62.1 & 68.5 & 52.5 & 36.7 & 54.3 & 39.5 & 37.8 & 50.0 \\
EAC-Net & 39.0 & 35.2 & 48.6 & 76.1 & 72.9 & 81.9 & 86.2 & 58.8 & 37.5 & 59.1 & 35.9 & 35.8 & 55.9 \\
DSIN & 51.7 & 40.4 & 56.0 & 76.1 & 73.5 & 79.9 & 85.4 & 62.7 & 37.3 & 62.9 & 38.8 & 41.6 & 58.9 \\
CMS & 49.1 & 44.1 & 50.3 & 79.2 & 74.7 & 80.9 & 88.3 & 63.9 & 44.4 & 60.3 & 41.4 & 51.2 & 60.6 \\
LP-Net & 43.4 & 38.0 & 54.2 & 77.1 & 76.7 & 83.8 & 87.2 & 63.3 & 45.3 & 60.5 & 48.1 & 54.2 & 61.0 \\
ARL & 45.8 & 39.8 & 55.1 & 75.7 & $\mathbf{7 7 . 2}$ & 82.3 & 86.6 & 58.8 & 47.6 & 62.1 & $\mathbf{4 7 . 4}$ & $\mathbf{5 5 . 4}$ & 61.1 \\
SCC & 48.2 & 50.3 & 55.8 & 79.9 & 73.6 & $\mathbf{8 8 . 1}$ & 89.9 & $\mathbf{6 8 . 7}$ & 42.2 & $\mathbf{6 5 . 2}$ & 41.6 & 42.0 & 62.1 \\
JÂA-Net & $\mathbf{5 3 . 8}$ & 47.8 & 58.2 & 78.5 & 75.8 & 82.7 & 88.2 & 63.7 & 43.3 & 61.8 & 45.6 & 49.9 & 62.4 \\
\hline \hline HiCOMEX & 50.2 & $\mathbf{5 5 . 3}$ & $\mathbf{5 8 . 2}$ & $\mathbf{7 9 . 9}$ & 73.8 & 86.9 & $\mathbf{9 0 . 7}$ & 66.8 & $\mathbf{4 5 . 5}$ & 62.4 & 46.1 & 48.7 & $\mathbf{6 3 . 7}$ \\
\hline
\end{tabular}

\section{Experiments}

\subsection{Dataset and evaluation metrics}

Our HiCOMEX is evaluated on two widely used datasets for assessing AU detection efficacy, i BP4D [22] and DISFA [10, in which both AU and landmark labels are provided.

BP4D contains 41 subjects, including 23 women and 18 men. Each involves eight sessions. There are 328 videos including about 140,000 frames with AU labels. 49 landmarks are provided for each frame. Akin to the setting of Zhao et al. 24, Li et al. 9], and Shao et al. [15, 12 AUs (1, 2, 4, 6, 7, 10, 12 14, 15, 17, 23, and 24) are assessed using subject exclusive three-fold cross-validation, where two folds are used for training and the remaining one is used for testing.

DISFA contains 27 video recordings from 12 women and 15 men, each has 4845 frames. Each frame is labelled with its $\mathrm{AU}$ intensity in the range from 0 to 5, and 66 landmarks are provided. According to the 
Table 2: F1-score(\%) in a comparative study of different state-of-the-art detection methods on the DISFA dataset. To ensure fairness in this, we did not list some results found using BP4D pre-training, e.g. JAA [15].

\begin{tabular}{c|c|c|c|c|c|c|c|c|c}
\hline \hline AU & 1 & 2 & 4 & 6 & 9 & 12 & 25 & 26 & Avg \\
\hline \hline LSVM & 10.8 & 10.0 & 21.8 & 15.7 & 11.5 & 70.4 & 12.0 & 22.1 & 21.8 \\
APL & 11.4 & 12.0 & 31 & 12.4 & 10.1 & 65.9 & 21.4 & 26.9 & 23.8 \\
DRML & 17.3 & 17.7 & 37.4 & 29.0 & 10.7 & 37.7 & 38.5 & 20.1 & 26.7 \\
EAC-Net & 41.5 & 26.4 & 66.4 & 50.7 & $\mathbf{8 0 . 5}$ & $\mathbf{8 9 . 3}$ & 88.9 & 15.6 & 48.5 \\
DSIN & 42.4 & 39.0 & 68.4 & 28.6 & 46.8 & 70.8 & 90.4 & 42.2 & 53.6 \\
CMS & 40.2 & 44.3 & 53.2 & $\mathbf{5 7 . 1}$ & 50.3 & 73.5 & 81.1 & 59.7 & 57.4 \\
LP-Net & 29.9 & 24.7 & 72.7 & 46.8 & 49.6 & 72.9 & 93.8 & 65.0 & 56.9 \\
ARL & 43.9 & 42.1 & 63.6 & 41.8 & 40.0 & 76.2 & $\mathbf{9 5 . 2}$ & $\mathbf{6 6 . 8}$ & 58.7 \\
\hline \hline HiCOMEX & $\mathbf{6 1 . 1}$ & $\mathbf{5 8 . 2}$ & $\mathbf{7 1 . 0}$ & 32.5 & 54.2 & 70.8 & 93.8 & 52.4 & $\mathbf{6 1 . 8}$ \\
\hline
\end{tabular}

Table 3: F1-score(\%) in an ablation study of HiCOMEX on the BP4D dataset.

\begin{tabular}{c|c|c|c|c|c|c|c|c|c|c|c|c|c}
\hline \hline AU & 1 & 2 & 4 & 6 & 7 & 10 & 12 & 14 & 15 & 17 & 23 & 24 & Avg \\
\hline \hline $\begin{array}{c}\text { HiCOMEX w/o intensity } \\
\text { distribution learning and } \\
\text { COMEX learning }\end{array}$ & 50.6 & 50.8 & 56.5 & 79.9 & $\mathbf{7 4 . 7}$ & $\mathbf{8 8 . 2}$ & 90.3 & $\mathbf{6 8 . 5}$ & 40.2 & $\mathbf{6 3 . 5}$ & 43.7 & 44.2 & 62.6 \\
\hline $\begin{array}{c}\text { HiCOMEX w/ only } \\
\text { COMEX learning (self-attention) }\end{array}$ & 51.1 & 51.7 & 59.0 & 78.0 & 73.4 & 84.9 & 89.9 & 64.5 & $\mathbf{5 0 . 8}$ & 61.9 & 47.2 & $\mathbf{4 8 . 8}$ & 63.4 \\
\hline $\begin{array}{c}\text { HiCOMEX w/ only } \\
\text { COMEX learning (Hopfield) }\end{array}$ & 52.1 & 53.8 & $\mathbf{6 0 . 7}$ & 76.3 & 73.3 & 87.1 & 90.1 & 64.0 & 45.1 & 63.3 & $\mathbf{4 8 . 5}$ & 45.6 & 63.3 \\
\hline $\begin{array}{c}\text { HiCOMEX w/ only intensity } \\
\text { distribution learning (LSTM) }\end{array}$ & $\mathbf{5 3 . 1}$ & 52.9 & 59.8 & 79.0 & 73.2 & 88.0 & 90.6 & 61.3 & 46.2 & 63.4 & 48.1 & 46.9 & 63.5 \\
\hline HiCOMEX & 50.2 & $\mathbf{5 5 . 3}$ & 58.2 & $\mathbf{7 9 . 9}$ & 73.8 & 86.9 & $\mathbf{9 0 . 7}$ & 66.8 & 45.5 & 62.4 & 46.1 & 48.7 & $\mathbf{6 3 . 7}$ \\
\hline
\end{tabular}

setting of Zhao et al. [24, Li et al. 9], and Shao et al. [15, an AU intensity equal to or greater than 2 is considered as an occurrence (others are treated as non-occurrences). Eight AUs (1, 2, 4, 6, 9, 12, 25, and 26) are evaluated.

It should be noted that in our experiments, we do not use any additional data or pre-trained models. That is to say, we only use BP4D and DISFA's own data for training and test.

Following the previous methods of Zhao et al. 24, Li et al. [9, and Shao et al. [15, F1-score (\%) are reported for comparison.

\subsection{Results and Discussions}

In this experiment, HiCOMEX is compared with several classical and state-of-the-art approaches, such as LSVM [6], JPML [23, DRML [24, CPM 21] EAC-Net 9], DSIN [3, CMS [14, LP-Net [1], ARL [16], SCC [7], and JÂA-Net [15].

Tables 1 and 2 lists the results obtained by our methods and almost all of the results from methods developed in the past three years. Compared with these baselines, HiCOMEX obtained an absolute advantage, once again surpassing the performance of state-of-the-art systems. HiCOMEX is (in absolute terms) $1.3 \%$ and $3.1 \%$ better with an F1-score that beats the previous state-of-the-art method, respectively on BP4D and DISFA datasets. Especially on AU2, AU10, AU12, and AU14, the performances are much better than the previous best method. These four AUs have many mutually interactive relationships with other AUs 8]. HiCOMEX uses this interactive relationship to strengthen the discriminative ability on AU2, AU10, and AU14 feature maps; our method still has absolute advantages compared with other SOTA graph-based methods, such as SCC [7] in Table 1.

\subsection{Ablation study}

In order to prove the effectiveness of the key modules in HiCOMEX, we undertook an ablation study in five settings: HiCOMEX without either an intensity distribution or COMEX learning, HiCOMEX with only intensity distribution learning (LSTM), HiCOMEX with only COMEX learning (self-attention), HiCOMEX 
Table 4: F1-score(\%) in an ablation study of HiCOMEX on the DISFA dataset.

\begin{tabular}{c|c|c|c|c|c|c|c|c|c}
\hline \hline AU & 1 & 2 & 4 & 6 & 9 & 12 & 25 & 26 & Avg \\
\hline \hline $\begin{array}{c}\text { HiCOMEX w/o intensity } \\
\text { distribution learning and } \\
\text { COMEX learning }\end{array}$ & 58.1 & 52.2 & 70.8 & 20.6 & 47.9 & 70.4 & 91.4 & $\mathbf{6 2 . 8}$ & 59.3 \\
\hline $\begin{array}{c}\text { HiCOMEX w/ only } \\
\text { COMEX learning (self-attention) }\end{array}$ & 60.1 & $\mathbf{5 9 . 2}$ & $\mathbf{7 1 . 3}$ & 26.5 & $\mathbf{5 5 . 3}$ & 71.5 & 93.8 & 53.1 & 61.4 \\
\hline $\begin{array}{c}\text { HiCOMEX w/ only } \\
\text { COMEX learning (Hopfield) }\end{array}$ & 61.0 & 57.2 & 70.6 & 32.4 & 51.4 & 70.7 & 90.7 & 51.1 & 60.6 \\
\hline $\begin{array}{c}\text { HiCOMEX w/ only intensity } \\
\text { distribution learning (LSTM) }\end{array}$ & 59.3 & 59.0 & 69.5 & $\mathbf{3 5 . 4}$ & 46.2 & $\mathbf{7 2 . 2}$ & $\mathbf{9 4 . 1}$ & 48.3 & 60.5 \\
\hline HiCOMEX & $\mathbf{6 1 . 1}$ & 58.2 & 71.0 & 32.5 & 54.2 & 70.8 & 93.8 & 52.4 & $\mathbf{6 1 . 8}$ \\
\hline
\end{tabular}

with only COMEX learning (Hopfield), and the complete HiCOMEX. Tables 3 and 4 list the results. Use of the BP4D intensity distribution and COMEX learning will boost the performance from $62.6 \%$ to $63.4 \%$ and $63.5 \%$ in terms of F1-score respectively compared with the HiCOMEX version without both an intensity distribution and COMEX learning. The full HiCOMEX pipeline performs best compared with all other versions. That means the local AU intensity distribution and COMEX relationship-learning modules can boost the performance. The effect of all local AU relationship-learning methods is to increase the F1-score of the pipeline shown in Fig. 3 without all relationship-learning modules by $1.1 \%$ on BP4D. The situation is similar to DISFA, the difference is that self-attention has the best effect.

\section{CONCLUSION}

We investigated the effectiveness of local AU feature map relationship modelling for AU detection. We propose the use of HiCOMEX to undertake AU recognition. Benefits from the strength of AU intensity distribution and COMEX relation learning, the best performance of HiCOMEX achieves the new state-ofthe-art of $63.7 \%$ and $61.8 \%$ F1-score on the public BP4D and DISFA datasets respectively: these scores are achieved without any use of any data or pre-trained models.

\section{References}

[1] Ba, J.L., Kiros, J.R., Hinton, G.E.: Layer normalization. arXiv preprint arXiv:1607.06450 (2016)

[2] Chu, W.S., De la Torre, F., Cohn, J.F.: Learning spatial and temporal cues for multi-label facial action unit detection. In: 2017 12th IEEE International Conference on Automatic Face \& Gesture Recognition (FG 2017). pp. 25-32. IEEE (2017)

[3] Corneanu, C., Madadi, M., Escalera, S.: Deep structure inference network for facial action unit recognition. In: Proceedings of the European Conference on Computer Vision (ECCV). pp. 298-313 (2018)

[4] Ekman, R.: What the face reveals: Basic and applied studies of spontaneous expression using the Facial Action Coding System (FACS). Oxford University Press, USA (1997)

[5] Ertugrul, I.Ö., Jeni, L.A., Cohn, J.F.: Pattnet: Patch-attentive deep network for action unit detection. In: BMVC. p. 114 (2019)

[6] Fan, R.E., Chang, K.W., Hsieh, C.J., Wang, X.R., Lin, C.J.: Liblinear: A library for large linear classification. Journal of machine learning research 9(Aug), 1871-1874 (2008)

[7] Fan, Y., Lam, J.C., Li, V.O.K.: Facial action unit intensity estimation via semantic correspondence learning with dynamic graph convolution. In: AAAI. pp. 12701-12708 (2020) 
[8] Li, G., Zhu, X., Zeng, Y., Wang, Q., Lin, L.: Semantic relationships guided representation learning for facial action unit recognition. In: Proceedings of the AAAI Conference on Artificial Intelligence. vol. 33, pp. 8594-8601 (2019)

[9] Li, W., Abtahi, F., Zhu, Z., Yin, L.: Eac-net: A region-based deep enhancing and cropping approach for facial action unit detection. In: 2017 12th IEEE International Conference on Automatic Face \& Gesture Recognition (FG 2017). pp. 103-110. IEEE (2017)

[10] Mavadati, S.M., Mahoor, M.H., Bartlett, K., Trinh, P., Cohn, J.F.: Disfa: A spontaneous facial action intensity database. IEEE Transactions on Affective Computing 4(2), 151-160 (2013)

[11] Niu, X., Han, H., Yang, S., Huang, Y., Shan, S.: Local relationship learning with person-specific shape regularization for facial action unit detection. In: Proceedings of the IEEE Conference on Computer Vision and Pattern Recognition. pp. 11917-11926 (2019)

[12] Ramsauer, H., Schäfl, B., Lehner, J., Seidl, P., Widrich, M., Gruber, L., Holzleitner, M., Pavlović, M., Sandve, G.K., Greiff, V., et al.: Hopfield networks is all you need. arXiv preprint arXiv:2008.02217 (2020)

[13] Reale, M.J., Klinghoffer, B., Church, M., Szmurlo, H., Yin, L.: Facial action unit analysis through 3d point cloud neural networks. In: 2019 14th IEEE International Conference on Automatic Face \& Gesture Recognition (FG 2019). pp. 1-8. IEEE (2019)

[14] Sankaran, N., Mohan, D.D., Setlur, S., Govindaraju, V., Fedorishin, D.: Representation learning through cross-modality supervision. In: 2019 14th IEEE International Conference on Automatic Face \& Gesture Recognition (FG 2019). pp. 1-8. IEEE (2019)

[15] Shao, Z., Liu, Z., Cai, J., Ma, L.: Jaa-net: Joint facial action unit detection and face alignment via adaptive attention. arXiv preprint arXiv:2003.08834 (2020)

[16] Shao, Z., Liu, Z., Cai, J., Wu, Y., Ma, L.: Facial action unit detection using attention and relation learning. IEEE Transactions on Affective Computing (2019)

[17] Srivastava, N., Hinton, G., Krizhevsky, A., Sutskever, I., Salakhutdinov, R.: Dropout: a simple way to prevent neural networks from overfitting. The journal of machine learning research 15(1), 1929-1958 (2014)

[18] Tu, C.H., Yang, C.Y., Hsu, J.Y.j.: Idennet: Identity-aware facial action unit detection. In: 2019 14th IEEE International Conference on Automatic Face \& Gesture Recognition (FG 2019). pp. 1-8. IEEE (2019)

[19] Vaswani, A., Shazeer, N., Parmar, N., Uszkoreit, J., Jones, L., Gomez, A.N., Kaiser, Ł., Polosukhin, I.: Attention is all you need. In: Advances in neural information processing systems. pp. 5998-6008 (2017)

[20] Wang, Z., Li, Y., Wang, S., Ji, Q.: Capturing global semantic relationships for facial action unit recognition. In: Proceedings of the IEEE International Conference on Computer Vision. pp. 3304-3311 (2013)

[21] Zeng, J., Chu, W.S., De la Torre, F., Cohn, J.F., Xiong, Z.: Confidence preserving machine for facial action unit detection. In: Proceedings of the IEEE international conference on computer vision. pp. $3622-3630$ (2015)

[22] Zhang, X., Yin, L., Cohn, J.F., Canavan, S., Reale, M., Horowitz, A., Liu, P., Girard, J.M.: Bp4dspontaneous: a high-resolution spontaneous $3 \mathrm{~d}$ dynamic facial expression database. Image and Vision Computing 32(10), 692-706 (2014)

[23] Zhao, K., Chu, W.S., De la Torre, F., Cohn, J.F., Zhang, H.: Joint patch and multi-label learning for facial action unit and holistic expression recognition. IEEE Transactions on Image Processing 25(8), 3931-3946 (2016) 
[24] Zhao, K., Chu, W.S., Zhang, H.: Deep region and multi-label learning for facial action unit detection. In: Proceedings of the IEEE Conference on Computer Vision and Pattern Recognition. pp. 3391-3399 (2016) 


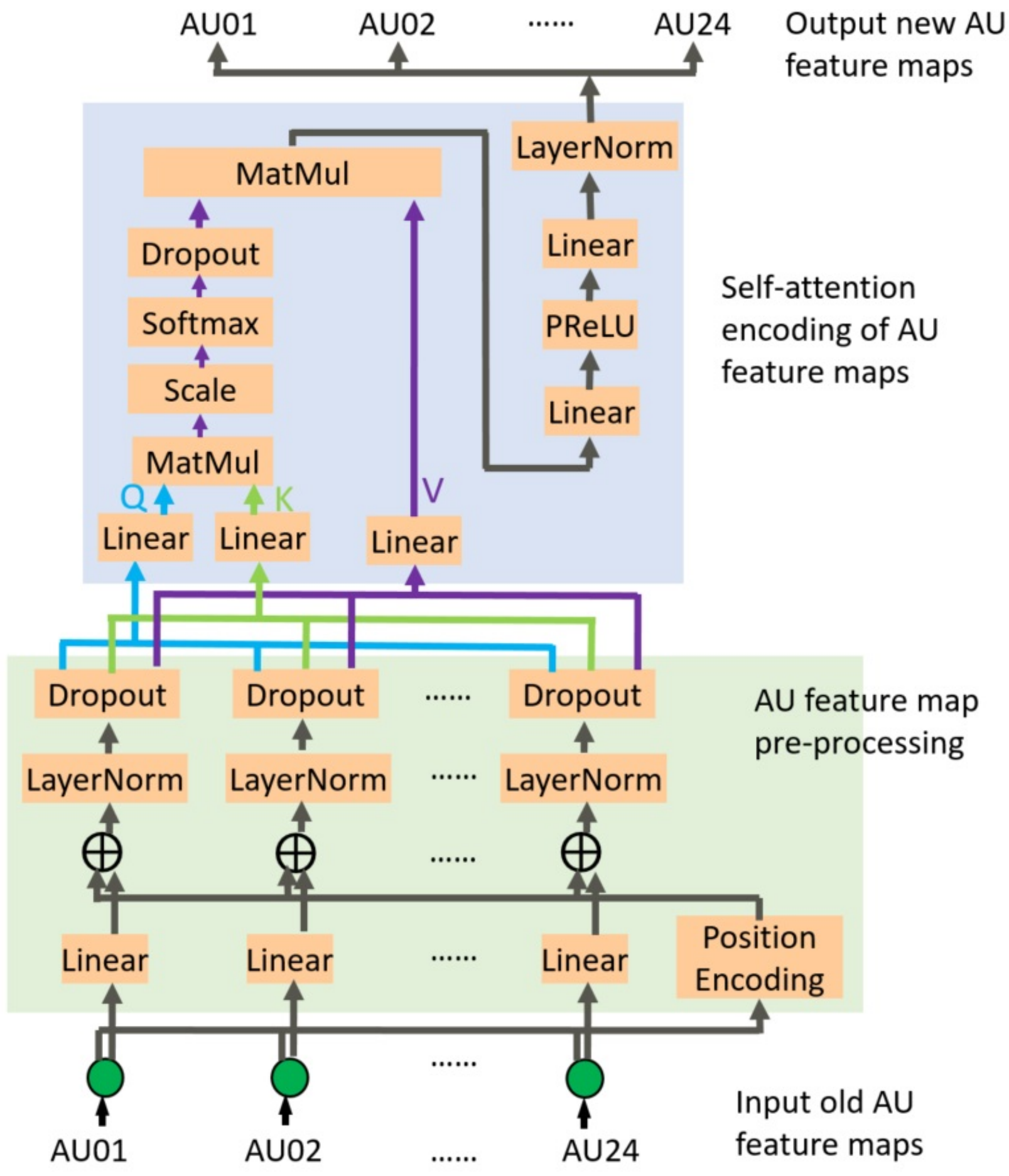

Figure 6: AU COMEX relation learning with self-attention encoding. The encoding is composed of two parts. The first is to perform the pre-processing of a linear transformation on the old AU feature maps, and in this process the coded position information of the AU feature maps are added. The second is the self-attention encoding layers, which can be described as mapping a query and a set of key-value pairs to an output, where the query, key, value, and output are all vectors. The output is calculated as a weighted sum of values, where the weight assigned to each value is derived by using the corresponding key query compatibility function. 


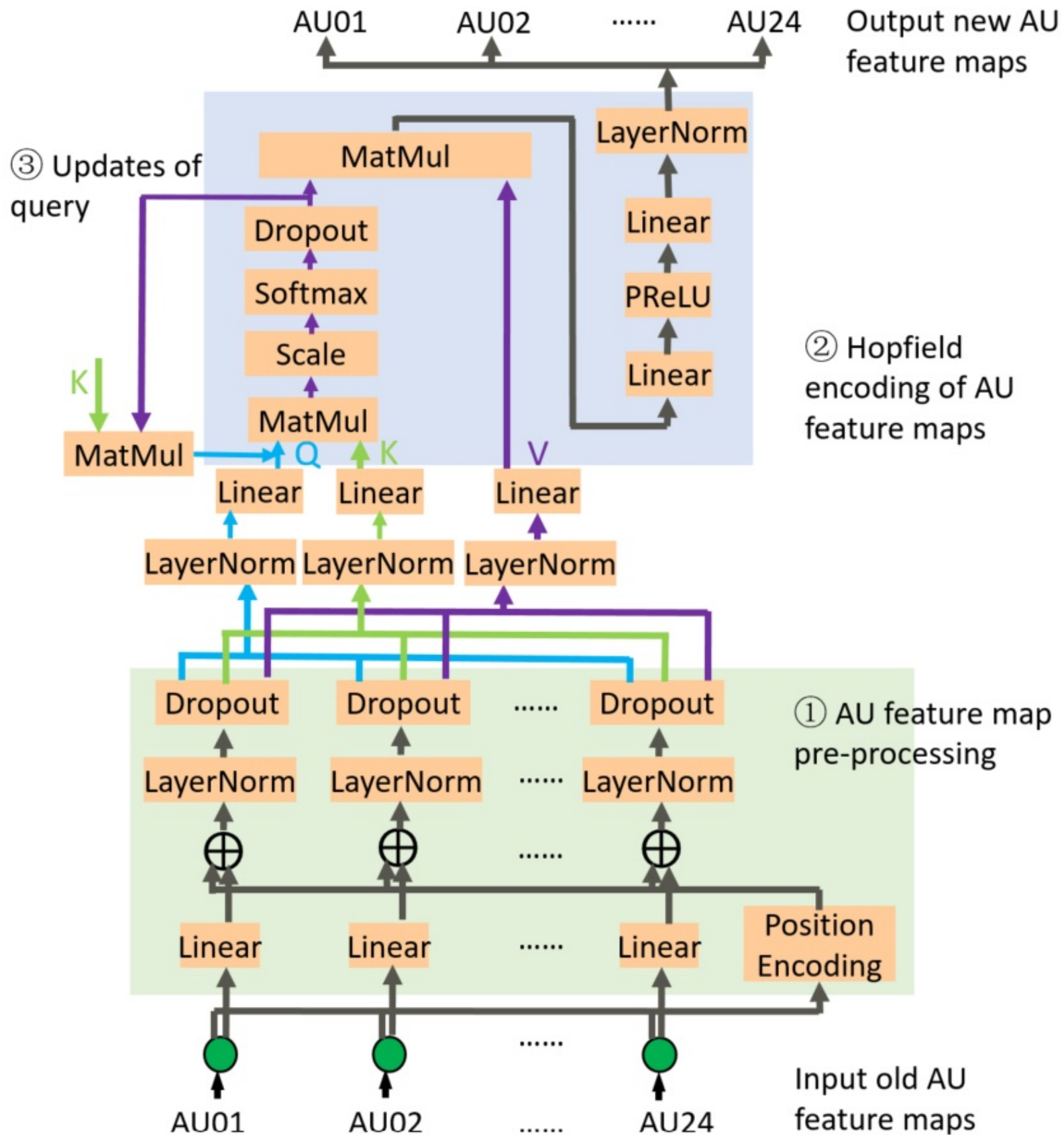

Figure 7: AU COMEX relationship learning with a Hopfield layer. The Hopfield relationship learning consists of three parts: 1)pre-processing of a linear transformation on the old AU feature maps, (in this process the coded position information of the AU feature maps is added); 2) self-attention; 3) updates of the query. 